\title{
Implementation of Standard Operating Procedure in Ophthalmic Practice during Covid 19 Era across Pakistan
}

\author{
Uzma Taqi $^{1}$, Erum Shahid ${ }^{2}$, Uzma Fasih ${ }^{3}$ \\ ${ }^{1}$ Baqai Medical University, ${ }^{2-3}$ Karachi Medical and Dental College \& Abbasi Shaheed Hospital, Karachi
}

\begin{abstract}
Purpose: To analyze the implementation of standard operating procedures (SOP) in different ophthalmic settings of Pakistan during Covid 19.
\end{abstract}

Study Design: Cross sectional online survey.

Place and Duration of Study: Baqai Medical University \& Karachi Medical and Dental College, from $11^{\text {th }}$ to $25^{\text {th }}$ January 2021.

Methods: This online survey included doctors working as consultants, medical officers and trainees in ophthalmology setups. A self-designed questionnaire on Google forms was sent online to see the implementation of standard operating procedures during COVID 19.

Results: A total of 400 participants were selected by snowball sampling technique. A response rate was 105 (26.5\%). Females were $66(62.86 \%)$. Among all participants, 36.19\% responded that they always screened patients for COVID 19 at reception. Doctors who always wore masks were $96.19 \%$. Out of those who responded, $16.19 \%$ and $11.43 \%$ always wore protective goggles and disposable gowns respectively. Only $55.24 \%$ practiced hand sanitization, $18.10 \%$ washed hands with soap and $30.48 \%$ cleaned slit lamps after seeing each patient. Doctors who always asked for PCR before ocular surgery under general anesthesia were $65(61.90 \%)$ and for local anesthesia were $36(34.29 \%)$. Direct Ophthalmoscopy, tonometry, and gonioscopy were performed when necessary by $56.19 \%, 80.00 \%, 80.00 \%$ of doctors respectively. Only $69.5 \%$ were satisfied with precautionary measures.

Conclusion: Clinic management needs improvement in implementing SOPs among patients in an eye OPD. Fifty percent of the ophthalmologists received PPE by their administration. Only $69.5 \%$ doctors were satisfied with precautionary measures.

Key Words: COVID 19, standard operating procedures, Pakistan, Ophthalmology

How to Cite this Article: Taqi U, Shahid E, Fasih U. Implementation of Standard Operating Procedure in Ophthalmic Practice During Covid 19 Era Across Pakistan. Pak J Ophthalmol. 2022, 38 (1): 36-42.

Doi: $10.36351 /$ pjo.v38i1.1307

Correspondence: Erum Shahid

Karachi Medical and Dental College \& Abbasi Shaheed Hospital, Karachi

Email:drerum007@hotmail.com

Received: June 24, 2021

Accepted: December 12, 2021

\section{INTRODUCTION}

The ongoing COVID-19 pandemic is a rapid rise catastrophic warning to human lives and livelihood all over the world. ${ }^{1}$ Severe acute respiratory syndrome coronavirus 2 (SARS-CoV-2) spreads primarily through person to person in close contact by droplets of saliva or discharge from the nose when they talk, cough or sneeze, by touching a virus containing 
surface or an object, and then touching the eyes, mouth or nose, ${ }^{2,3}$ or by aerosol droplets of the conjunctiva in case of conjunctivitis. ${ }^{4}$ As of January 22, 2021, 219 countries and territories have been affected around the world. Reported registered cases were 99,538,884 and a mortality rate of $2.14 \%$ with $2,134,449$ deaths had been recorded. ${ }^{5}$ It has spread like bush fire with tremendous pressure on the healthcare system. Healthcare workers are a part of the front line, which are fighting against the pandemic. ${ }^{6}$ According to PAHO Director Carissa F. Etienne, large number of healthcare workers have been infected with COVID19 virus and have died globally. By September 2020, over 10,000 healthcare workers got infected with coronavirus in Africa. ${ }^{7}$ In Washington D.C., by September 02, 2020, nearly 570,000 health workers have contracted the virus and more than 2,500 could not make their way out and died. ${ }^{8}$ There were 3387 healthcare workers in China, who got sick with this virus and 23 passed away. ${ }^{9}$

The first two cases of Coronavirus were confirmed on February 26th, 2020 in Pakistan. By January 22, 2021, 532,412 cases of COVID-19 were recorded and 11,295 succumbed to this virus in Pakistan. At least, 10,300 health professionals have been infected and amid them, 100 have died. ${ }^{10}$ Khyber Pakhtunkhwa reported the highest number of infections among health care workers at 2,638, followed by 2,463 in Sindh and 2,534 in Punjab. ${ }^{11}$

However, Ophthalmologists are one of the most high-risk medical specialties as they are very close to the patients during examination. Slit-lamp is an extremely necessary tool for an eye examination in which the distance between the patient and ophthalmologist is less than 12 inches. Furthermore, there is no distance between the examiner and the patient during direct ophthalmoscopy. ${ }^{12}$ The ocular secretions and tears could be responsible for the spread of virus. Dr. Li Wenliang, MD a 33-year old ophthalmologist working in Wuhan, China, was the first doctor who raised the alarm about the novel coronavirus, the first ophthalmologist who contracted this virus by an asymptomatic glaucoma patient and died. $^{13}$

This study was conducted to see the implementation of standard operating procedures (SOP) in various ophthalmic settings all over Pakistan during the COVID-19 era. It will help to identify the flaws in our health care system and also let the ophthalmologists be aware of the importance of SOPs.
Moreover, it will be beneficial for policy makers and stakeholders to facilitate the implementation of standard operating procedures in ophthalmic departments of various hospitals and clinics to curb virus among health care workers and patients.

\section{METHODS}

This cross-sectional survey was conducted online in the ophthalmology department of Baqai Medical University \& Karachi Medical and Dental College. The study was approved by the Institutional Ethics Committee of Baqai medical university, hospital. All the doctors working as consultants, medical officers, trainees and residents in ophthalmology clinics, ophthalmology departments of government and private hospitals all over the country during the last 6 months of Covid-19 lockdown, were invited to participate in the study, from $11^{\text {th }}$ to $25^{\text {th }}$ January 2021.

A self-designed questionnaire in English language was used on Google forms to collect the data. The form was shared via e-mail and WhatsApp link to the participants. The sampling technique was the snowball technique. We accepted responses for 2 weeks. Participation and completion of the survey were regarded as informed consent of the respondents. The survey and the participants were kept anonymous. Participants provided demographic data including age range, gender, qualification, place of practice, and designation. Incomplete forms were excluded from the analysis.

The study questionnaire consisted of 23 multiple choice questions. Other than demographic details, there were 15 questions based on standard operating procedures (SOP) selected from the guidelines provided by the Indian ophthalmological society. ${ }^{14}$ Among them 3 questions were related to the administration, whether the patients were screened at the reception of the hospital by asking questions related to symptoms of COVID-19 and history of travel. Whether they were seated with social distancing in the waiting area of the clinic and the patients had worn face mask were also asked. Seven questions were about the participating doctors; (wearing a face mask, washing hands with soap, cleaning with a sanitizer, using a breath shield on the slit lamp, wearing protective goggles or face shield, gown, and cleaning slit lamp after each patient). The responses were classified into every time, frequently, often, or never. The next 3 questions were about 
performing diagnostic procedures including direct ophthalmoscopy, tonometry, and gonioscopy (always, never, and when mandatory in a patient for an eye examination). Two questions were about practicing polymerase chain reaction (PCR) testing before ophthalmic surgeries under local and general anesthesia (always, frequently, often, or never). The last two questions were about the personal protective equipment (PPE) provided to the doctors by their hospital or arranged by themselves and were they satisfied with the precautions they were taking.

Data was compiled on Microsoft Excel. Frequencies were generated for the categorical variables and means for numerical variables.

\section{RESULTS}

A total of 400 participants were requested to fill the questionnaire online. One hundred and five (105) participants responded. Participants with missing data were excluded. The response rate of complete data was 26.25\%. Data analysis was done on 105 participants. In this study, 39 (37.14\%) participants were males and $66(62.86 \%)$ were females. Their ages ranged between 21 to 80 years, which were further categorized in decades (Table 1). Most of the respondents, (35.24\%) were in the age group of $41-50$ years. Table 2 shows the details of responses received by the participants. Approximately 22\% never asked screening questions for COVID-19. Only $1(0.95 \%)$ responded that patients never wore masks. However, majority of the participants (96.19\%) always wore masks and only $16.19 \%$ and $11.43 \%$ always wore protective goggles and disposable gowns respectively. More than half of the responders sanitized their hands after examining each patient (55.24\%). Doctors who always ordered PCR for COVID-19 for local anesthesia were 36 $(34.29 \%)$ and for general anesthesia were 65 $(61.90 \%)$.

The highest number of participants, who performed direct ophthalmoscopy, tonometry, and gonioscopy when necessary were, 56.19\%, 80.00\%, and $80.00 \%$ respectively while only $3.81 \%$ never did tonometry during Pandemic.

Only $51 \%$ were provided PPE by the hospital. Sixty one percent of the participants were satisfied by the SOP.

Table 1: Demographic Features of the Participants in Percentages.

\begin{tabular}{|c|c|}
\hline Variables & n $(\%)$ \\
\hline \multicolumn{2}{|l|}{ Gender } \\
\hline Males & $39(37.14 \%)$ \\
\hline Females & $66(62.86 \%)$ \\
\hline \multicolumn{2}{|l|}{ Age Range } \\
\hline $21-30$ years & $14(13.33 \%)$ \\
\hline $31-40$ years & $31(29.52 \%)$ \\
\hline $41-50$ years & $37(35.24 \%)$ \\
\hline $51-60$ years & $19(18.10 \%)$ \\
\hline $61-70$ years & $4(3.81 \%)$ \\
\hline $71-80$ years & $0(0.00 \%)$ \\
\hline \multicolumn{2}{|l|}{ Qualification } \\
\hline FCPS/FRCS & $79(75.24 \%)$ \\
\hline MCPS & $3(2.86 \%)$ \\
\hline MS & $2(1.90 \%)$ \\
\hline MBBS & $21(20.00 \%)$ \\
\hline \multicolumn{2}{|l|}{ Province } \\
\hline Sindh & $53(50.48 \%)$ \\
\hline Punjab & $37(35.24 \%)$ \\
\hline Islamabad & $4(3.81 \%)$ \\
\hline Balochistan & $4(3.81 \%)$ \\
\hline Khyber Pakhtun Khawa & $6(5.71 \%)$ \\
\hline Kashmir & $1(0.95 \%)$ \\
\hline \multicolumn{2}{|l|}{ Place of Work } \\
\hline Urban & $99(94.29 \%)$ \\
\hline Rural & $6(5.71 \%)$ \\
\hline \multicolumn{2}{|l|}{ Place of Work } \\
\hline Government Hospital & $58(55.24 \%)$ \\
\hline Private Hospital & $36(34.29 \%)$ \\
\hline Private Clinic & $11(10.47 \%)$ \\
\hline \multicolumn{2}{|l|}{ Designation } \\
\hline Consultant & $83(79.05 \%)$ \\
\hline Resident/Trainee & $10(9.52 \%)$ \\
\hline Medical Officer & $12(11.43 \%)$ \\
\hline House Officer & $7(6.67 \%)$ \\
\hline
\end{tabular}

FCPS: Fellow of College of Physicians and Surgeons. FRCS: Fellow of Royal College of Surgeons.

MCPS: Member of College of Physicians and Surgeons. MS: Master of Surgery.

MBBS: Bachelor of Medicine and Bachelor of Surgery. 
Implementation of Standard Operating Procedure in Ophthalmic Practice during Covid 19 Era

Table 2: Percentages of Implementation of SOPS in Various Ophthalmic Practices Across Pakistan.

\begin{tabular}{lcccc}
\hline Questions & Always (\%) & Frequently (\%) & Often (\%) & Never (\%) \\
\hline Patient is screened at reception & $38(36.19 \%)$ & $20(19.05 \%)$ & $24(22.86 \%)$ & $23(21.90 \%)$ \\
Patient wears mask & $54(51.43 \%)$ & $35(33.33 \%)$ & $15(14.29 \%)$ & $1(0.95 \%)$ \\
Patient is seated with social distancing in waiting area & $23(21.90 \%)$ & $28(26.67 \%)$ & $30(28.57 \%)$ & $24(22.86 \%)$ \\
Do you wear a mask & $101(96.19 \%)$ & $3(2.86 \%)$ & $0(0.00 \%)$ & $1(0.95 \%)$ \\
Do you wear protective goggles/ face shield & $17(16.19 \%)$ & $10(9.52 \%)$ & $34(32.38 \%)$ & $44(41.90 \%)$ \\
Do you wear a disposable gown in OPD & $12(11.43 \%)$ & $11(10.48 \%)$ & $26(24.76 \%)$ & $56(53.33 \%)$ \\
Do you sanitize your hands after examining each patient & $58(55.24 \%)$ & $29(27.62 \%)$ & $16(15.25 \%)$ & $2(1.90 \%)$ \\
Do you wash hands with soap after seeing each patient & $19(18.10 \%)$ & $35(33.33 \%)$ & $40(38.10 \%)$ & $11(10.48 \%)$ \\
Do you clean slit lamp after every patient & $32(30.48 \%)$ & $31(29.52 \%)$ & $23(21.90 \%)$ & $19(18.10 \%)$ \\
Do you use slit lamp breath shield & $99(94.29 \%)$ & - & - & $6(5.71 \%)$ \\
Do you screen patient before local surgery by PCR & $36(34.29 \%)$ & $15(14.29 \%)$ & $8(7.62 \%)$ & $46(43.81 \%)$ \\
Do you screen patient before general surgery by PCR & $65(61.90 \%)$ & $12(11.43 \%)$ & $7(6.67 \%)$ & $21(20.00 \%)$ \\
\hline
\end{tabular}

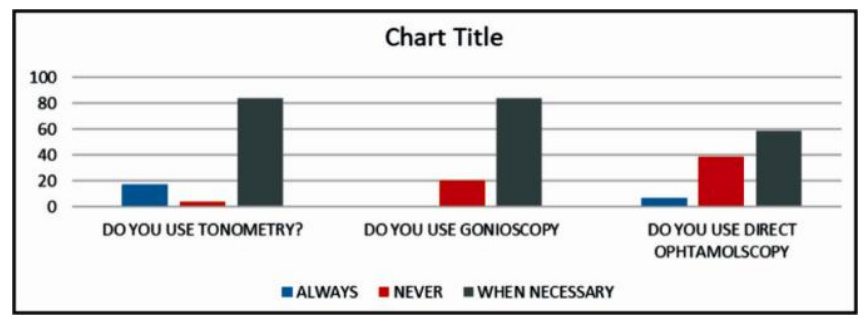

Figure 1: Percentages of participants using various diagnostic procedures during COVID 19.

\section{DISCUSSION}

Ophthalmology practice is distinctive, as it requires close contact during patient examination, routine usage of reusable equipment, and many highly touch surfaces, which results in a high risk of disease transmission among ophthalmology patients. We have broadly categorized the SOP into clinic management and staff protection for ease of understanding.

In our study, $21.90 \%$ of patients presenting for an eye examination at an ophthalmology clinic were never asked about the screening questions at the reception and $22.86 \%$ of them were never seated with social distance. Patients coming to an eye outpatient department always wore a mask represented by $51.43 \%$ of the total. It has been recommended by the American Academy of ophthalmology that every ophthalmology clinic must be equipped with screening stations and patients should be screened before entering the waiting area. ${ }^{15} \mathrm{~A}$ detailed history of respiratory symptoms, fever, recent travel from a highrisk area, and in contact with a family member back from COVID 19 outbreak zones, is important. On the basis of this, patients have been further categorized into low, medium, or high-risk patients. Patients with urgent ophthalmic problems either a medium risk or high risk should be immediately attended by an ophthalmologist with full PPE to decrease the time spent at the clinic. COVID-19 patients with urgent ophthalmic problems can be attended following local hospital guidelines and taking precautions. ${ }^{16}$ Waiting areas of the outpatient departments (OPD) should be kept as empty as possible and patient seated with a minimum distance of 2 meters or 6 feet apart. ${ }^{17}$ The statistics of our clinic setups specify that SOP's are not rigorously followed in hospitals and there is no accountability by higher authorities.

In our study, $96.19 \%$ of the ophthalmologists always wore face masks but only $16.19 \%$ wore protective goggles or face shields and $11.43 \%$ wore protective gowns while attending patients in eye clinics. This could be because of a lack of awareness or shortage of disposable supplies by the hospital administration. It is important not only to provide PPE to the health worker but also provide instructions on how to don and remove PPE to decrease the risk of infection. $^{18}$

The importance of hand hygiene is highlighted during the Pandemic outbreak and every healthcare personnel should follow guidelines provided by WHO using alcohol-based sanitizer or washing hands with soap after every patient encounter. ${ }^{19}$ Use of sanitizer after every patient was adopted by $55.24 \%$ and washing hand with soap by $18.10 \%$ of the doctors in our study.

Direct ophthalmoscopy, tonometry and gonioscopy were performed in every patient by $6.67 \%$, $16.19 \%$ and $0.95 \%$ of the ophthalmologists respectively. Tonometer tips should be disinfected with $70 \%$ alcohol or disposable tonometer tip should be used. However, the best option is the use of noncontact tonometer. ${ }^{20}$ This option is not widely available especially in small clinics and in rural areas 
of our country. Instead of slit lamp fundus examination, ophthalmologist should consider using indirect ophthalmoscopy. ${ }^{21}$

Specialties including ophthalmologists, anesthesiologists, and otorhinolaryngologists are prone to get infections as they are exposed to aerosolized particles of respiratory droplets and conjunctival secretions. $^{22}$ Tracheal intubation and manual ventilation cause a 3 to 7 folds increase in the respiratory tract infection in a health care worker. Ophthalmologist in Pakistan that never screen their patients with COVID PCR testing before ocular surgeries under local anesthesia was $43.81 \%$ and $20.00 \%$ never screened their patients before surgery under general anesthesia. Selected public hospitals in our country have been providing PCR testing for COVID-19 free of cost. This test is quite expensive in private setups of our country (7000-10,000 PKR or 62.26 US dollars) and is not accessible for everyone. These factors bound doctors to continue with their surgical practices without PCR testing, jeopardizing their own safety. However, it should be kept in mind that elective surgery and laser treatment patients should be deferred in the outbreak of Pandemic. ${ }^{22}$ If surgery is required, proper PPE should be worn by all the operation theatre staff and the surgeon.

Ophthalmologists in Pakistan, who have to arrange for PPE themselves, were $51.43 \%$. The private practice of ophthalmologists has been hugely affected by the pandemic, the surgical burden has been reduced. Many doctors have become jobless and their pay has been reduced. In the presence of all these factors, buying your own PPE is an extra burden and it should be taken care of by the hospital administration or government. It has been reported that lack of PPE increases stress and anxiety level among health care professionals. $^{23}$

Fortunately, our country did not experience the disaster of severe acute respiratory syndrome (SARS) epidemic in 2003. This is the time we should learn a lesson and be prepared for the challenges that might come in future. It is highly recommended for the competent authorities and the stakeholders to make sure that all the leading hospitals and independent private clinics are following SOPs to prevent the spread of COVID-19 among our patients, staff, and ophthalmologist. The government should also provide financial assistance to the hospitals and ophthalmologists working in independent eye clinics or hospitals for their PPE including N95 masks, protective goggles or headgear, disposable gowns, sanitizers, and breath-shields on the slit lamp. Health care workers should be trained vigilantly for appropriate use of PPE, protocols regarding clinic flow and instrument cleaning. COVID19 diagnostic kit should be widely and readily available so that PCR testing can be routinely done for every ophthalmic surgery under local and general anesthesia. This is the era, where the significance of artificial intelligence and telemedicine should be emphasized globally. Furthermore, third world countries like Pakistan cannot afford to lose a qualified ophthalmologist, because it takes years to reach a competency level.

The limitation of our study is the low response rate to the survey resulting in a small sample size. Secondly, data gathered from rural areas of Pakistan is very scarce, as the accessibility of the internet is inadequate in these areas. Large data gathered equally from rural and urban areas would have different results. Thirdly self-reporting surveys have their own biases as an interpretation by the participants is different.

The strength of the study is that it represents data from Pakistan about the standard operating procedures in ophthalmic practice that are being followed by the ophthalmologist and doctors working in eye hospitals and clinics. The study gives us insight into improving our daily ophthalmic practice. It points out the domains of Standard operating procedures that are lacking and need implementation.

\section{CONCLUSION}

Clinic management needs improvement in implementing SOPs among patients in an eye OPD. Fifty percent of the ophthalmologists received PPE by their administration. Only $69.5 \%$ doctors were satisfied with precautionary measures.

\section{Ethical Approval}

The study was approved by the Institutional review board/Ethical review board (BMU-EC/02-2021).

\section{Conflict of Interest}

Authors declared no conflict of interest. 


\section{REFERENCES}

1. Eredem H, Lucey D. Healthcare worker infections and deaths due to COVID-19: A survey from 37 nations and a call for WHO to post national data on their website. Int J Infect Dis. 2021; 102: 239-241.

Doi: $10.1016 /$ j.ijid.2020.10.064.

2. CDC. How coronavirus spreads. Available: https://www.cdc.gov/coronavirus/2019ncov/prepare/transmission.html [Accessed 27 Mar 2020].

3. Lu CW, Liu XF, Jia ZF. 2019-nCoV transmission through the ocular surface must not be ignored. Lancet (London, England), 2020; 395 (10224): e39.

4. Xia J, Tong J, Liu M, Shen Y, Guo D. Evaluation of coronavirus in tears and conjunctival secretions of patients with SARS-CoV-2 infection. J Med Virology, 2020; 92 (6): 589-594.

5. Covid -19 coronavirus pandemic 2020 Dec 10 . www.worldometers.info>coronavirus

6. Buchtele N, Rabitsch W, Knaus HA, Wohlfarth P. Containment of a traceable COVID - 19 outbreak among healthcare workers at a hematopoietic stem cell transplantation unit. Bone Marrow Transplantation, 2020; 55 (7): 1491-1492.

7. African Regional Office (AFRO)/World Health Organization (WHO/OMS) 2020. Over 10,000 Health Workers in Africa Infected with COVID-19. [cited 2020 Jul 23]. Available from:

https://www.afro.who.int/news/over-10-000-healthworkers-africa-infected-covid-19.

8. Pan American Health Organization (PAHO)/World Health Organization (WHO) 2020. COVID - 19 has Infected Some 570.000 Health Workers and Killed 2,500 in the Americas. [cited 2020 Sep 27]. Available from: https://www.paho.org/en/news/2-9-2020-covid19-has-infected-some-570000-health-workers-andkilled-2500-americas-paho.

9. Zhan M, Qin Y, Xue X, Zhu S. Death from Covid-19 of 23 Health Care Workers in China. N Engl J Med. 2020; 382 (23): 2267-2268.

10. Pakistan latest victim of coronavirus. https://www.geo.tv/latest/274482-pakistan-confirmsfirst. Accessed 7 Mar 2020.

11. Death toll of healthcare workers in Pakistan due to COVID - 19 reaches 100 Read more At:

https://www.aninews.in/news/world/asia/death-toll-ofhealthcare-workers-in-pakistan-due-to-covid-19reaches-10020201201185445/

12. Adhi MI. Special situations Novel coronavirus disease pandemic and ophthalmologist perspective. J Pak Med Assoc. 2020; 70: 5.

13. Li JO, Shantha J, Wong TY, Wong EY, Mehta J, Lin H, et al. Preparedness among ophthalmologists: during and beyond the COVID-19 pandemic. Ophthalmology, 2020; 5: 569-572.
14. Sengupta S, Honavar SG, Sachdev MS, Sharma N, Kumar A, Ram J, Shetty R, Rao GS, Ramasamy K, Khanna R, Jain E. All India Ophthalmological Society-Indian Journal of Ophthalmology consensus statement on preferred practices during the COVID-19 pandemic. Indian J Ophthalmol. 2020; 68 (5): 711.

15. Important coronavirus updates for ophthalmologists. Available at: https://www.aao.org/headline/alertimportant-coronavirus-context(2020) accessed; 25 November 2021.

16. Liu W, Tang F, Fang LQ, De Vlas SJ, Ma HJ, Zhou JP, Looman CW, Richardus JH, Cao WC. Risk factors for SARS infection among hospital healthcare workers in Beijing: a case control study. Trop Med Intern Health, 2009; 14: 52-59.

17. Lai TH, Tang EW, Chau SK, Fung KS, Li KK. Stepping up infection control measures in ophthalmology during the novel coronavirus outbreak: an experience from Hong Kong. Graefe's Arch Clin Exp Ophthalmol. 2020; 258 (5): 1049-1055.

18. Boyce JM, Havill NL, Lipka A, Havill H, Rizvani R. Variations in hospital daily cleaning practices. Infection control and hospital epidemiology, 2010 Jan. 1; 31 (1): 99.

19. Kampf G, Todt D, Pfaender S, Steinmann E. Persistence of coronaviruses on inanimate surfaces and their inactivation with biocidal agents. J Hospital Infect. 2020; 104 (3): 246-251.

20. Romano MR, Montericcio A, Montalbano C, Raimondi R, Allegrini D, Ricciardelli G, Angi M, Pagano L, Romano V. Facing COVID-19 in ophthalmology department. Current Eye Research, 2020; 45 (6): 653-658.

21. Wan KH, Huang SS, Young AL, Lam DS. Precautionary measures needed for ophthalmologists during pandemic of the coronavirus disease 2019 (COVID-19).

22. Lim LW, Yip LW, Tay HW, Ang XL, Lee LK, Chin CF, Yong V. Sustainable practice of ophthalmology during COVID-19: challenges and solutions. Graefe's Arch Clin Exp Ophthalmol. 2020; 258 (7): 1427-1436.

23. Dong L, Bouey J. Public mental health crisis during COVID-19 pandemic, China. Emerging infectious diseases, 2020; 26 (7): 1616.

Doi: 103201/eid2607200407.

\section{Authors' Designation and Contribution}

Uzma Taqi; Assistant Professor: Concepts, Design, Literature Search, Data Acquisition, Data Analysis, Statistical Analysis, Manuscript Preparation, Manuscript Editing, Manuscript Review. 
Erum Shahid; Assistant Professor: Concepts, Design, Literature Search, Data Acquisition, Data Analysis, Statistical Analysis, Manuscript Preparation, Manuscript Editing, Manuscript Review.
Uzma Fasih; Associate Professor: Data analysis, Manuscript Preparation, Manuscript Editing, Manuscript Review.

\section{………}

